University of Nebraska - Lincoln

DigitalCommons@University of Nebraska - Lincoln

\title{
Structured decision making as a conceptual framework to identify thresholds for conservation and management
}

Julien Martin

Michael C. Runge

James D. Nichols

Bruce C. Lubow

William L. Kendall

Follow this and additional works at: https://digitalcommons.unl.edu/usgsstaffpub

Part of the Geology Commons, Oceanography and Atmospheric Sciences and Meteorology Commons, Other Earth Sciences Commons, and the Other Environmental Sciences Commons

This Article is brought to you for free and open access by the US Geological Survey at DigitalCommons@University of Nebraska - Lincoln. It has been accepted for inclusion in USGS Staff -- Published Research by an authorized administrator of DigitalCommons@University of Nebraska - Lincoln. 


\title{
Structured decision making as a conceptual framework to identify thresholds for conservation and management
}

\author{
Julien Martin, ${ }^{1,2,4}$ Michael C. Runge, ${ }^{2}$ James D. Nichols, $^{2}$ Bruce C. Lubow, $^{3}$ and William L. Kendall ${ }^{2}$ \\ ${ }^{1}$ Florida Cooperative Fish and Wildlife Research Unit, University of Florida, Gainesville, Florida 32611-0485 USA \\ ${ }^{2}$ Patuxent Wildlife Research Center, United States Geological Survey, 12100 Beech Forest Road, Laurel, Maryland 20708 USA \\ ${ }^{3}$ Natural Resource Ecology Laboratory, Colorado State University, Fort Collins, Colorado 80523 USA
}

Abstract. Thresholds and their relevance to conservation have become a major topic of discussion in the ecological literature. Unfortunately, in many cases the lack of a clear conceptual framework for thinking about thresholds may have led to confusion in attempts to apply the concept of thresholds to conservation decisions. Here, we advocate a framework for thinking about thresholds in terms of a structured decision making process. The purpose of this framework is to promote a logical and transparent process for making informed decisions for conservation.

Specification of such a framework leads naturally to consideration of definitions and roles of different kinds of thresholds in the process. We distinguish among three categories of thresholds. Ecological thresholds are values of system state variables at which small changes bring about substantial changes in system dynamics. Utility thresholds are components of management objectives (determined by human values) and are values of state or performance variables at which small changes yield substantial changes in the value of the management outcome. Decision thresholds are values of system state variables at which small changes prompt changes in management actions in order to reach specified management objectives. The approach that we present focuses directly on the objectives of management, with an aim to providing decisions that are optimal with respect to those objectives. This approach clearly distinguishes the components of the decision process that are inherently subjective (management objectives, potential management actions) from those that are more objective (system models, estimates of system state). Optimization based on these components then leads to decision matrices specifying optimal actions to be taken at various values of system state variables. Values of state variables separating different actions in such matrices are viewed as decision thresholds. Utility thresholds are included in the objectives component, and ecological thresholds may be embedded in models projecting consequences of management actions. Decision thresholds are determined by the above-listed components of a structured decision process. These components may themselves vary over time, inducing variation in the decision thresholds inherited from them. These dynamic decision thresholds can then be determined using adaptive management. We provide numerical examples (that are based on patch occupancy models) of structured decision processes that include all three kinds of thresholds.

Key words: adaptive resource management; ecological thresholds; patch occupancy models; stochastic dynamic programming; structured decision making.

\section{INTRODUCTION}

Thresholds and their relevance to conservation have become a popular topic of discussion among ecologists, conservation biologists, managers and policy makers (Burgman 2005, Bestelmeyer 2006). Unfortunately, in many cases the lack of a clear conceptual framework for thinking about thresholds may have led to confusion in attempts to apply the concept of thresholds to conservation decisions. Here, we advocate a framework for thinking about thresholds in terms of a structured decision making process (SDM; Clemen and Reilly

Manuscript received 5 February 2008; revised 1 August 2008; accepted 25 September 2008. Corresponding Editor: J. Belnap.

4 E-mail: julienm@ufl.edu
2001). The purpose of this framework is to promote a logical and transparent process for making informed decisions for conservation and management. Specification of such a framework leads naturally to consideration of definitions and roles of different kinds of thresholds in the process.

We distinguish among three kinds of thresholds that we believe to be relevant to making decisions in conservation: ecological, utility, and decision thresholds. Ecological thresholds can be defined generally as values of system state variables across which small changes produce either changes in system dynamics of specified magnitude (typically large or ecologically substantial changes) or changes to specific values of system-dynamic descriptive metrics (e.g., $P($ extinction $)=1)$. This 
operational definition is generally consistent with discussions of ecological thresholds encountered in the literature in which such thresholds are often defined as a point or a zone at which there is a sudden change in the condition of a biological system (e.g., system state; Fahrig 2001, Huggett 2005, Pascual and Guichard 2005, Groffman et al. 2006, Bennetts et al. 2007). For example, in the case of vegetation communities in the Chihuahuan Desert, the ecological threshold may occur when modest changes in precipitation patterns produce a shift from grass- to shrub-dominated communities (Brown et al. 1997, Groffman et al. 2006). Another example is the case of extinction thresholds in metapopulation systems. The threshold occurs when a small change in some parameter (e.g., proportion of habitat in a landscape that is suitable) triggers a change in the equilibrium occupancy from some positive probability to 0 (Lande 1987, Fahrig 2001, Benton 2003). Ecological thresholds may also be defined based on movement of systems to permanent absorbing states rather than to strictly transient states. This can have important consequences for making conservation decisions. Indeed, conservation decisions may be very different if a small change in an environmental variable shifts the system from a transient state (e.g., high abundance of a species) to another transient state (e.g., local extinction but with possibility of recolonization of the system) rather than to a permanent absorbing state (e.g., global extinction with no possibility of recolonization). Other concepts related to ecological thresholds, such as ecological resilience (Holling 1973) and elasticity (Bodin and Wiman 2007), can also have important implications for making optimal decisions. Ecological resilience can be defined as the magnitude of perturbation a system can absorb before it changes from one state to another (Gunderson 2000), whereas elasticity is the time required for a system to return to its equilibrium state after a perturbation (Bodin and Wiman 2007). There is now widespread consensus among ecologists that ecological thresholds and other related concepts (e.g., resilience and elasticity) are relevant to both science and management (Bodin and Wiman 2007). However, in order for ecological thresholds (and related concepts) to be most useful to natural resource management and conservation, we believe that they must be incorporated into models that are then used to derive management decisions (see also Conroy et al. 2003, Bestelmeyer 2006).

Two other kinds of thresholds are relevant to natural resource management and conservation decision making. We define utility thresholds as values of state or performance variables at which small changes yield substantial changes in the value of the management outcome. For example, we might specify that an objective of management of a particular species in a national park is that the population size should remain high enough for park visitors to have a reasonable chance to observe the species during a visit (let's say $10 \%)$. The park managers may have determined that this population size is about $N^{*}$. Therefore, according to this management objective, management should seek to keep the population size above $N^{*}$ individuals. Unlike ecological thresholds, which are part of the pattern and process of nature, utility thresholds are subjective and determined by human values. In some cases, however, the development of utility thresholds may be based on ecological thresholds. For instance, if managers are concerned about preventing a species from going extinct, then in this case $N^{*}$ may be determined based on extinction thresholds and the desire to keep population size far above such a threshold. But even in this latter scenario, the decision to focus on preventing the extinction of a particular species is subjective and based on human values.

Finally, we define decision thresholds (sometimes referred to as management thresholds, see Bennetts et al. 2007) based on values of system state variables that should prompt specific management actions. Decision thresholds are thus conditional on, and derived from, ecological and utility thresholds. For example, if one state variable (e.g., water levels in a wetland) is influenced directly by management actions (e.g., irrigation) and is known to affect another state variable (the proportion of patches occupied by a species, $\psi$ ), the decision threshold corresponds to the values of water levels and $\psi$ at which a small change will prompt a change in management action (e.g., from no irrigation to some irrigation) in order to achieve specified management objectives (e.g., $\psi>0.3$ see Numerical example 1 for more details).

We believe that discussions of thresholds in the ecological literature have not always been clear, and that distinctions among types of thresholds have not always been adequate. For example, it is very common to find no distinction in practice between utility and decision thresholds. A common approach to management under the declining-population paradigm (Caughley 1994) is to view a finite rate of increase $(\lambda)$ of 1 simultaneously as a utility and a decision threshold. A declining population $(\lambda<1)$ is viewed as undesirable, such that $\lambda=1$ is a utility threshold. The decision process entails testing for a negative trend in abundance (e.g., based on empirical data and statistical models), with a "significant" negative trend then triggering management actions (decision threshold). Management under the SDM approach that we advocate (see below) tends to produce decision thresholds that are more conservative than this trend-detection approach. If $\lambda=1$ is our utility threshold, then management actions typically occur well before the population is actually declining, in an effort to keep $\lambda \geq 1$. Indeed, the trenddetection approach has been criticized as leading to unnecessary delays in management actions (Maxwell and Jennings 2005, Nichols and Williams 2006). In addition, the usual approach of placing trend detection in a hypothesis-testing framework invites discussion about type I and II error rates (e.g., arbitrary $\alpha$ for 
hypothesis testing) and the relative risks associated with these errors (see Field et al. 2004 for a discussion of this topic).

The approach to structured decision making that we advocate herein focuses directly on the objectives of management, with an aim to providing decisions that are optimal with respect to those objectives, given existing knowledge about system behavior. Such an approach clearly distinguishes the components of the decision process that are inherently subjective (management objectives, potential management actions) from those that are more objective (models of system behavior, estimates of system state) (Williams et al. 2002, Nichols and Williams 2006). Utility thresholds may be included as components of management objectives, and ecological thresholds may be embedded in models projecting consequences of management actions. Optimization based on these components then leads directly to decision matrices specifying optimal actions to be taken at various values of system state variables. Values of state variables separating different actions in such matrices are viewed as decision thresholds. These decision thresholds are neither arbitrary nor subjective, but are derived from the management objectives, available actions and system models. It is through this process that utility and ecological thresholds together yield decision thresholds.

Our primary goals in this paper are to advocate the SDM process for conservation problems and to clarify the roles of the three classes of thresholds within this process. The presentation is structured into four sections. First, we describe an approach to establish decision thresholds based on a SDM framework. Second, we show how these decision thresholds are not equivalent to, but are modified by, changes in utility thresholds. Third, we identify sources of uncertainty that can influence decision thresholds and discuss methods to account for these uncertainties. Fourth, we show how learning about ecological thresholds can be achieved through SDM. Finally, we discuss benefits of using SDM to identify decision thresholds for conservation. Our presentation includes numerical examples that illustrate our points and descriptions of analytical methods that can be applied to implement the SDM approach.

\section{Using SDM to Establish Decision Thresholds}

SDM is a formal method for analyzing a decision by breaking it into components (Clemen and Reilly 2001). This approach helps to identify the impediments to a decision, and to focus effort on the appropriate component(s). The goal is then to identify the optimal decision in terms of the specified objectives. SDM is rooted in decision theory, which provides a powerful framework for making decisions about the management of complex systems (Bellman 1957, Intriligator 1971, Williams et al. 2002, Burgman 2005, Halpern et al. 2006). Examples of useful applications of SDM can be found in a variety of fields, including engineering, economics and natural resource management (e.g., Johnson et al. 1997, Clemen and Reilly 2001, Miranda and Fackler 2002, Halpern et al. 2006). In the context of conservation, the elements of the decision making process often include the following components: objectives, potential management actions, model(s) of system behavior (in particular models that predict how system states change with different management options), a monitoring program to keep track of the system state and finally a method to identify the solution (Williams et al. 2002, Dorazio and Johnson 2003, McCarthy and Possingham 2007). Two of these components, model(s) and estimates of system state, are typically characterized by substantial uncertainties that must be accommodated in the optimization process.

\section{Objectives and management decisions}

The specification of objectives is a critical component of any decision-making process. Objectives correspond to what the relevant stakeholders strive to achieve via the implementation of management actions. Objectives constitute the basis for assessing alternative decisions, where the "success" of a decision to meet the management objectives serves as a way to evaluate the decision options (Clemen and Reilly 2001, Conroy and Moore 2001). Conservation objectives, and to a lesser extent, potential management actions, are typically based on value judgments of the stakeholders (Nichols and Williams 2006). Examples of objectives relevant to conservation include maximizing species diversity in a natural area or minimizing the probability of quasiextinction of a threatened species (Kendall 2001). In order to take into consideration the concerns of all relevant stakeholders involved in the decision process, it is often appropriate to include utility thresholds that can be viewed as constraints on an objective. For instance, in the example presented below we envision a situation in which stakeholders wish to maximize the release of water for irrigation, while maintaining a specified proportion of wetlands occupied by a species of special interest (the utility threshold). The objectives and associated constraints should generally be determined through discussions among stakeholders (Kendall 2001). Formal techniques to narrow down and select appropriate objectives may sometimes be helpful (see Clemen and Reilly 2001, Burgman 2005). Once objectives and constraints have been selected, they can be formalized mathematically into an objective function. The objective function quantifies the benefit (or return) obtained by implementing specific decisions at each time step, accumulated over the time horizon of the decision problem (Lubow 1995, Williams et al. 2002, Fonnesbeck 2005).

\section{Models of system behavior}

In contrast to management objectives and potential management actions, which are inherently subjective 
components of the decision process, models of system behavior and measures of confidence in the models should preferably be based on a scientific approach (Nichols 2001, Nichols and Williams 2006). The model (or models) of system behavior projects the consequences of potential management decisions at time $t\left(d_{t}\right)$ on the system (e.g., shift from one state at time $t, x_{t}$, to another state at time $\left.t+1, x_{t+1}\right)$. Information about ecological thresholds can be incorporated into models of system behavior (see Numerical example 1).

Models provide a basis for predicting system response to management actions (e.g., Lubow 1995, Williams et al. 2002). For example, the equation

$$
x_{t+1}=F\left[x_{t}, d\left(x_{t}\right)\right]
$$

describes a Markov process. That is, the probability distribution of the system state at $t+1$ depends on the system state at $t$ and the decision at $t$. Discrete Markov decision models can be analyzed with dynamic programming methods to find the optimal decision at each time step (e.g., the decision that maximizes the return; Miranda and Fackler 2002, Burgman 2005).

\section{Analytical method to identify optimal decisions}

The Hamilton-Jacobi-Bellman (HJB) algorithm describes a method for solving a stochastic dynamic programming problem (Williams et al. 2002):

$V_{t}^{*}\left(x_{t}\right)=\max _{d_{t}}\left[U\left(d_{t} \mid x_{t}\right)+\sum_{x_{t+1}} P\left(x_{t+1} \mid x_{t}, d_{t}\right) \times V_{t+1}^{*}\left(x_{t+1}\right)\right]$.

where $U\left(d_{t} \mid x_{t}\right)$ specifies the current return derived from taking decision $d_{t}$ when the system is in state $x_{t}$, and $P\left(x_{t+1} \mid x_{t}, d_{t}\right)$ corresponds to the transition probabilities to the system state at $t+1\left(x_{t+1}\right)$, given the current state $\left(x_{t}\right)$ and decision $\left(d_{t}\right)$ at time $t$. These transition probabilities are obtained from the state dynamic function (or model of system behavior, see Eq. 1). $V_{t}^{*}$ is the optimal future value function given $x_{t}$ and assuming that the optimal sequence of decisions is followed. The optimal sequence of decisions can be obtained in principle by repeated recursive applications of Eq. 2, (see Miranda and Fackler 2002, Williams et al 2002, for a description on how to solve the HJB equation).

Decision thresholds, then, arise out of the derived optimal strategy, as values of state variables across which the optimal decision changes. When more than one system state variable is considered, decision matrices can be constructed (see Example 1 and Fig. 2 for a graphical representation of a decision matrix). Next, we illustrate the SDM framework to derive decision thresholds with a simple hypothetical example that focuses on a conservation problem. Part of the example presented below was inspired by Miranda and Fackler (2002).

\section{Numerical example 1}

Water from a large, heterogeneous wetland is used to irrigate agricultural areas and is also necessary for the persistence of a species of special interest (hereafter referred to as species A). The wetland contains $L_{t}$ units of water at the beginning of year $t$, and $R_{t}$ units are released for irrigation during year $t ; p_{t}$ units of rain replenish the wetland annually. The wetland can only hold a maximum of $K$ units of water $(K=2000$ in our example). Any surplus of water flows out without any costs or benefits. The proportion of suitable habitat patches within the wetland occupied by species $A$ in year $t+1, \psi_{t+1}$, is influenced by $L_{t}$, because $L_{t}$ governs local probabilities of patch extinction and patch colonization for the species.

The stakeholders' goal is to maximize water use for economic benefits, but with the constraint that at least $30 \%$ of suitable habitat patches will be occupied by species $\mathrm{A}$. The value of $30 \%$ is based on human values, and could be determined for example by the desire of managers to maintain the species at historical levels (i.e., before the wetland was affected by human activities). Specifically, 0.3 is a utility threshold, and we greatly devalue any decision about irrigation, $d_{t}$, that yields an expected occupancy less than 0.3 in the next year (i.e., $\hat{\psi}_{t+1}<0.3$, where $\hat{\psi}_{t+1}$ is the expected probability of a suitable patch being occupied in year $t+1$, given $\psi_{t}$ and $d_{t}$ ). A policy that maximizes the economic benefits while allowing for the persistence of species $\mathrm{A}$ in at least $30 \%$ of suitable habitat, can be determined by using Stochastic Dynamic Programming (SDP) (Bellman 1957, Lubow 1995).

As noted above, this example is highly simplified and is not intended to be particularly realistic. For example, we assume that the state variables (i.e., $\psi_{t}$ and $L_{t}$ ) are estimated without errors, which is unlikely in reality. However, incorporation of this source of uncertainty adds substantially to the complexity of the example (Williams and Nichols 2001) and would detract from our main goal of illustrating our conceptual framework for thinking about thresholds.

\section{Utility function}

The utility function for this problem expresses the current return or utility associated with the decision to irrigate $I$ units of water,

$$
U_{t}\left(x_{t}=L_{t}, d_{t}=I_{t}\right)= \begin{cases}0, & \hat{\psi}_{t+1}<0.3 \\ R_{t}, & \hat{\psi}_{t+1} \geq 0.3\end{cases}
$$

with

$$
R_{t}=\min \left(K, I_{t}\right) .
$$

This equation indicates that given that the system state at time $t$ is $L_{t}$ and that decision $I_{t}$ is made at time $t$, the utility is equal to $R_{t}$ if $\hat{\psi}_{t+1} \geq 0.3$ and 0 otherwise. For this problem, the set of management actions is the number of water units allocated to irrigation, expressed 
in increments of 100 units, with a maximum release of 2000 units (i.e., $I_{t} \in\{0,100,200, \ldots, 2000\}$ ). The quantity $R_{t}$ cannot be greater than the sum of $L_{t}$ and $p_{t}$. The utility threshold is expressed as a constraint (i.e., $\hat{\psi}_{t+1} \geq 0.3$ ) in the utility function and is based on value judgment. Of course, in a more realistic scenario one would have to probably consider more complex utility functions. For instance, one may want to consider the fact that the irrigation needs go down as rainfall goes up. However, for the purpose of illustration we kept our problem simple.

\section{Models of system behavior}

To solve our decision problem we need to keep track of two state variables, $L$ (water level) and $\psi$ (patch occupancy). The dynamics for the amount of water in the wetland are described by

$$
L_{t+1}=L_{t}+p_{t}-R_{t}
$$

where $p_{t}$, which corresponds to the precipitation between times $t$ and $t+1$, was included in the model as a normally distributed random variable (with mean $=$ 550 and $\mathrm{SD}=104$ ).

The model for the dynamics of patch occupancy is

$$
\psi_{t+1}=\psi_{t} \times\left(1-\varepsilon_{t}\right)+\left(1-\psi_{t}\right) \times \gamma_{t}
$$

where $\varepsilon$ and $\gamma$ are patch extinction and colonization parameters, respectively (MacKenzie et al. 2006). We assume the following relationship between $\gamma$ and $L$ :

$$
\gamma_{t}= \begin{cases}0, & \text { if } L_{t}<T \\ 0.1, & \text { if } L_{t} \geq T\end{cases}
$$

where $T$ is an ecological threshold, below which $\gamma$ drops dramatically (Fig. 1a). Thus, information about the ecological threshold is incorporated into the model of system behavior. Note that in a real case study the patch occupancy models described in this section could be developed and evaluated with empirical data by following the methods presented in MacKenzie et al. (2006).

We assume the following linear-logistic relationship between $\varepsilon$ and $L$ :

$$
\varepsilon_{t}=\frac{1}{1+\exp \left(-\alpha-\beta \times L_{t}\right)}
$$

where $\alpha$ and $\beta$ are, respectively, the intercept and slope of the logistic function.

We view the response of patch extinction presented in Fig. $1 \mathrm{~b}$ as a threshold response because of the notion that a small change in values $L$ can induce a large change in $\varepsilon$ (see Farhig 2001, Huggett 2005, Bennetts et al. 2007). However, for $\varepsilon$ the ecological threshold is not as clearly defined as for $\gamma$. Indeed, there is a range of values of $L$ that could trigger an abrupt response in $\varepsilon$ (as opposed to a single value in the case of $\gamma$ ). Thus, the area between the dotted lines in Fig. 1b illustrates that ecological thresholds can be viewed as a range of values.
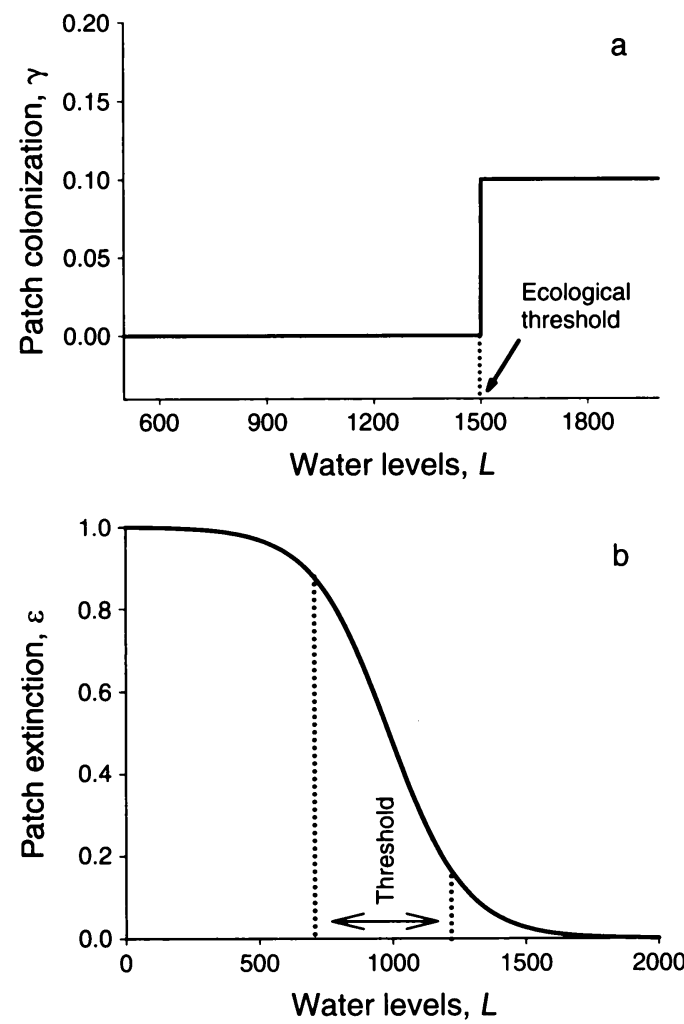

FIG. 1. Relationships between water levels and (a) patch colonization and (b) patch extinction of species $\mathrm{A}$ under Model 1.

In fact, the bounds of this range of values were determined subjectively (i.e., by visual examination of the curve). We realize that often there is some subjectivity involved when defining a response as a threshold response (e.g., how steep should the relationship be before it can be viewed as an ecological threshold), and we leave it up to each investigators to define particular ecological responses in their study systems as ecological thresholds or not. The key point is that ecological models may or may not include functional relationships that are viewed as thresholds. The SDM framework accommodates such relationships and can be used to learn about their functional forms (see Threshold and adaptive management).

\section{Establishing decision thresholds}

The optimal value for the objective function satisfies the recurrence relationship specified in the HJB equation:

$$
\begin{aligned}
V_{t}^{*}\left(x_{t}=L_{t}\right)=\max _{d_{t}}[ & U_{t}\left(x_{t}=L_{t}, d_{t}=I_{t}\right) \\
& +\sum_{x_{t+1}} P\left(x_{t+1}=L_{t+1} \mid x_{t}=L_{t}, d_{t}=I_{t}\right) \\
& \left.\times V_{t+1}^{*}\left(L_{t+1}\right)\right] .
\end{aligned}
$$



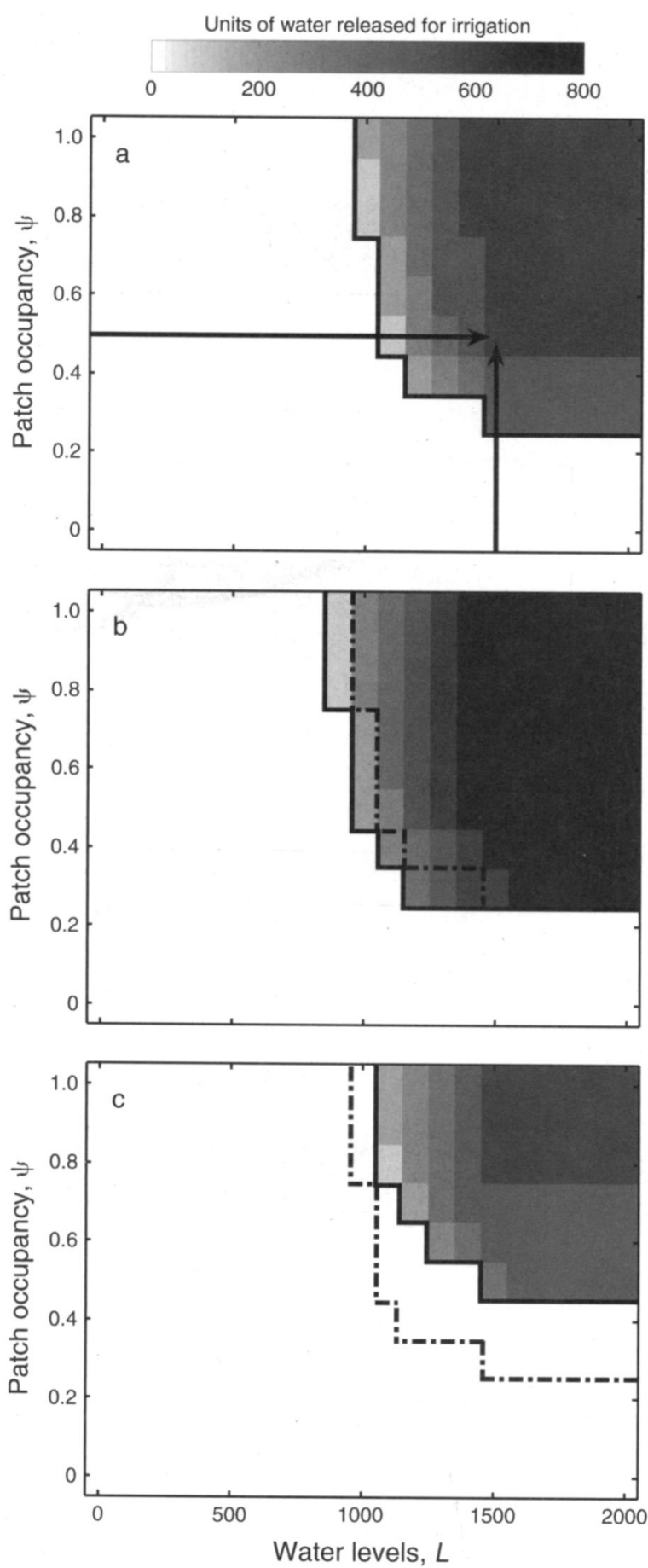

FIG. 2. Plots of optimal irrigation decisions as a function of water levels $\left(L_{t}\right)$ and patch occupancy $\left(\psi_{t}\right)$ of species A. (a) Ecological threshold for patch colonization $(T)$ was set at $T=$ 1500 units of water, and (b) $T=800$ units of water, with the utility threshold set at 0.3 in both cases. (c) Utility threshold was set at 0.5 and $T=1500$. The shades of gray correspond to the amount of water released for irrigation (from 0 water units [lighter shade] to 800 units [darker shade]). Decision thresholds are represented by these changes in shading. The thick black lines indicate the decision thresholds at the boundary between some irrigation and no irrigation. Dot-dashed lines in panels (b) and (c) indicate shifts in decision thresholds when compared to
We approximated the decision problem for an infinite time horizon via backward iteration dynamic programming, by iterating through time steps until a stable policy was maintained for 100 time periods, using program ASDP Version 3.2 (Lubow 2001). Fig. 2a shows the optimal irrigation decisions for a given level of patch occupancy and quantity of water. For instance, if in a given year $(t) \psi_{t}=0.5$ and $L_{t}=1500$ units, the optimal decision is to irrigate $I_{t}=600$ units during year $(t)$. The thick lines in Fig. 2a indicate the decision thresholds. As expected the decision thresholds in Fig. 2a indicate that more water can be released for irrigation as more water units are present in the wetland and as more patches become occupied. In addition, except at high water levels, the decision threshold between irrigation and no irrigation occurs at occupancy levels above the utility threshold of 0.3 (unless the impoundment is quite full).

As noted above, decision thresholds are derived from all of the components of the decision process, including utility thresholds and any ecological thresholds that may be incorporated into the system models. In order to illustrate the relationship between ecological and decision thresholds we conducted an analysis in which we shifted the ecological threshold for patch colonization ( $T$ was set to 800 instead of 1500 in Eq. 6). Hereafter, we refer to the model with $T=1500$ as Model 1 and to the model with $T=800$ as Model 2. Fig. 2b shows that this shift in the ecological thresholds induced a shift in the resulting decision thresholds. For instance, for most values of $\psi$, the decision threshold at the boundary between no irrigation and some irrigation occurred at lower water levels when the ecological threshold was set to 800 (Fig. 2b). We also increased the utility threshold from 0.3 to 0.5 (in Eq. 3) in order to observe the resulting change in the decision threshold (Fig. 2c). In this latter scenario, for most values of $\psi$, the boundary between no irrigation and some irrigation occurred at higher water levels than when the utility threshold was set at 0.3 (Fig. 2c).

Fig. 3a shows the trajectory of $\psi$ over time assuming that optimal irrigation policies (presented in Fig. 2a) are followed. The initial drop in $\psi$ below the utility threshold reflects transient dynamics associated with the initial conditions (Fig. 3). As expected, $\psi$ rarely fell below the utility threshold of 0.3 (the value specified as a constraint in the objective function), and indeed is much larger most of the time (Fig. 3a). In this sense, the optimal strategy is far more conservative (with respect to maintaining $\psi \geq 0.3$ ) than an approach of waiting until $\psi$, approaches 0.3 and only then limiting irrigation. In contrast, if all the water from the wetland was systematically released for irrigation, $\psi$ would rapidly approach 0 . Fig. $3 \mathrm{~b}$ also shows the evolution of $\psi$ over

panel (a). Black arrows point to the irrigation policy at time $t$ : 600 units of water, when $\psi$ at $t$ is 0.5 and water level at $t$ is 1500 units of water. 
time assuming that optimal irrigation policies for Model 1 are followed but assuming that the utility threshold was raised to 0.5 (i.e., $\hat{\psi}_{t+1} \geq 0.5$, see Eq. 3). Again, $\psi_{t}$ rarely fell below the utility threshold (the value of $\psi_{t}$ averaged over 30000 iterations was 0.66 , whereas it was 0.46 when the utility threshold was set to 0.3 , see Table 1 , scenarios 1 and 3). However, when simulating the average amount of water devoted to irrigation under the optimal policy, this quantity was greater when the utility threshold was set at $0.3(504 \pm 84$ units of water irrigated per year [mean \pm SD], Table 1, scenario 1) than when it was set at 0.5 (487 \pm 55 units of water irrigated per year, Table 1, scenario 3). To maintain occupancy above 0.5 , the wetland needs to be kept at a higher level; but this carries the risk that sometimes precipitation that could have been used for irrigation is lost when the wetland overflows, thus the long-term average water withdrawals are lower. In other words, the behavior of the optimal strategy can be explained in the following way: the long-term average rate of withdrawal can be no more than the average annual precipitation, but to take full advantage of this precipitation, the wetland should be kept far from capacity, so no water is ever lost to overtopping. The constraint in the objective (i.e., $\psi \geq$ specified value) works in the opposite direction, however, by requiring a fuller wetland. The optimal strategy balances these two competing objectives.

We now consider how the approach described above would perform when compared to a more typical decision process by presenting some simulation results for a hypothetical scenario of a more "typical" process (hereafter denoted as TYP, this is just one scenario among many possibilities). Assume that instead of following the SDM approach, managers decided to irrigate 550 units of water (which corresponds to the average amount of annual rainfall) when $\psi_{t}>0.3$ and 0 units of water when $\psi_{t} \leq 0.3$. Thus, in this scenario managers do not distinguish between utility and decision thresholds. We used Model 1 as the model of system behavior to compare the results for both approaches. The results for the simulation of $\psi$ for scenario TYP are presented in Fig. 3c, and show that $\psi_{t}$ fell below the utility threshold more often than when using SDM (compare Fig. 3a and 3c). The average annual irrigation for the TYP is also smaller $(\bar{I}=409$ units of water per year) than when using the SDM approach $(I=504$ units of water per year; see scenario 1 in Table 1 ).

\section{SOURCES OF UNCERTAINTY THAT INFLUENCE DECISION THRESHOLDS}

Uncertainty is an important component of any decision making process (Williams et al. 1996, Burgman 2005, Halpern et al. 2006). Accounting for uncertainty in the decision process will often influence the resulting decision thresholds. There are several types of uncertainty that can influence decisions and associated thresholds. One of them, environmental stochasticity, affects almost every natural system. It can result from
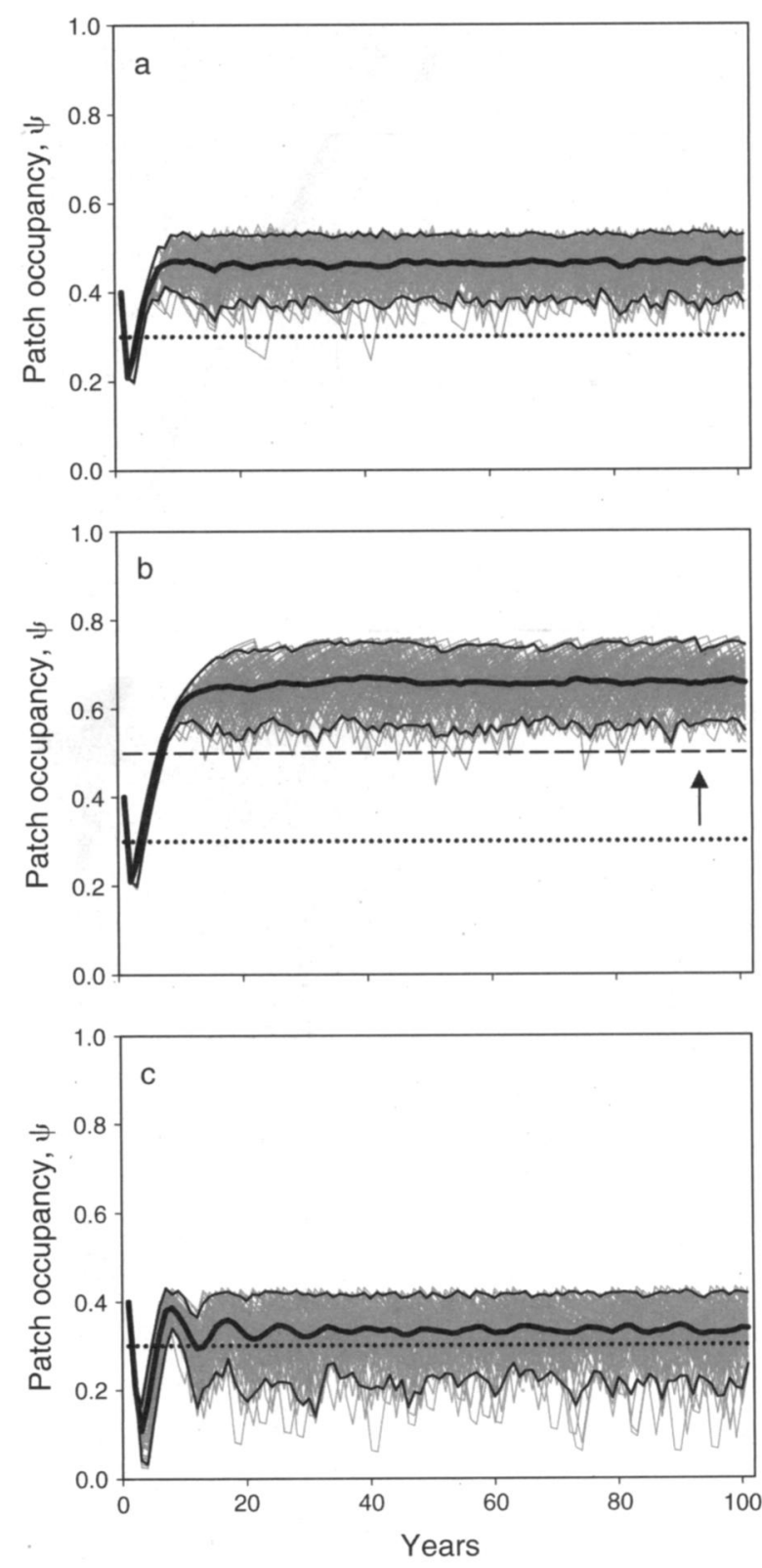

FIG. 3. Simulation (100 runs) of patch occupancy $(\psi)$ of species A over time (100 years) when optimal irrigation policies are followed. Each simulation run is represented by a gray line, and the mean (at each time step over the 100 runs) is represented by a thick black line. Thin black lines correspond to $2.5 \%$ and $97.5 \%$ quantiles. (a) Scenario 1: optimal irrigation decisions were derived with a utility threshold set at 0.3 (dotted line). (b) Scenario 2: optimal irrigation decisions were derived with a utility threshold set at 0.5 (dashed line). (c) Scenario 3: irrigation decisions were based on a hypothetical scenario (TYP) in which managers irrigate 550 units of water when patch occupancy is greater than 0.3 but stop irrigating when patch occupancy falls below 0.3 . The initial conditions for the simulations were set at $\psi=0.4$ and initial water levels $=1000$ units. 
TABLE 1. Results from simulations of average annual patch occupancy $(\psi)$ of species A, water levels $(\bar{L})$, and irrigation $(\bar{I})$ over time (30000 iterations) when irrigation policies derived from stochastic dynamic programming (SDP) are followed.

\begin{tabular}{cclrrrl}
\hline \hline Scenario number & Utility threshold & $\operatorname{SD}(\beta)$ & $T$ & $\psi$ & $\bar{I}$ \\
\hline 1 & 0.3 & 0.0 & 1500 & $0.46(0.04)$ & $504(81)$ & $1440(87)$ \\
2 & 0.3 & 0.0 & 800 & $0.43(0.05)$ & $540(124)$ & $1290(111)$ \\
3 & 0.5 & 0.0 & 1500 & $0.66(0.06)$ & $487(55)$ & $1480(68)$ \\
4 & 0.3 & 0.001 & 1500 & $0.51(0.11)$ & $475(82)$ & $1490(90)$ \\
5 & 0.3 & 0.001 & 800 & $0.50(0.11)$ & $507(105)$ & $1420(112)$ \\
6 & 0.5 & 0.001 & 1500 & $0.64(0.10)$ & $422(116)$ & $1560(119)$
\end{tabular}

Notes: Results for six scenarios are reported. Each scenario differed in at least one of the following categories: specified utility threshold; the value of $T$ (i.e., the ecological threshold for patch colonization in Eq. 6); the process variation (standard deviation, $\mathrm{SD}(\beta)$ associated with parameter $\beta$ in Eq. 7). Numbers inside parentheses correspond to the standard deviations.

variation in weather patterns or from unrecognized variation in habitat structure (Williams et al. 1996, 2002). Environmental uncertainty can be incorporated into the models as process variance (temporal or spatial), using random variables with specified distributions. In Numerical example 1, environmental stochasticity entered the model by modeling annual rainfall as a normally distributed random variable (mean $=550$, SD $=104$, where the standard deviation reflects the process variance). In order to illustrate the fact that environmental stochasticity can substantially influence decision thresholds we incorporated some process variance in model parameter $\beta$ (in Eq. 7 , we set $\operatorname{SD}(\beta)=0.001$ ), reflecting unidentified environmental factors that influence the relationship between water level and patch extinction probability (e.g., variation in food resources for species A). Optimal decisions based on this revised model are shown in Fig. 4a. Incorporating this additional source of uncertainty led to more conservative irrigation policies (i.e., for most values of the state space, less water was allocated to irrigation, see Fig. 4a and compare with Fig. 2a) than when $\beta$ was assumed to be deterministic (i.e., $\operatorname{SD}(\beta)=0$ ). Indeed, the amount of water devoted to irrigation annually, averaged over 30000 iterations, was reduced when some process variance was associated with parameter $\beta$ (see Table 1 , $\bar{I}$ for scenario 1 was greater than for scenario 4 ; $\bar{I}$ for scenario 2 was greater than for scenario 5 ; and $\bar{I}$ for scenario 3 was greater than for scenario 6). Values of $\psi_{t}$ averaged over 30000 iterations were greater for scenarios 4 and $5(\operatorname{SD}(\beta)=0.001)$ than for scenario 1 and 2 $(\operatorname{SD}(\beta)=0$, Table 1$)$. However, $\psi$ was slightly greater for scenario $3(\operatorname{SD}(\beta)=0)$ than for scenario $6(\operatorname{SD}(\beta)=$ 0.001, Table 1).

Process variation can be estimated from historical data through analytical methods that separate it from sampling variance (see Gould and Nichols 1998, Burnham and White 2002). The process variance associated with modeled parameters allows for the incorporation of multiple sources of environmental stochasticity without having to necessarily explicitly model each source of variation individually. For instance, if a factor is not directly affected by management decisions and is difficult to measure, its effect on system behavior can simply be incorporated as an unidentified component of a global process variance that includes effects from several factors. In fact, in order to derive decision thresholds based on SDP, it is frequently advisable to model explicitly only the variables that are directly affected by management decisions (or alternatively that are assumed to have a large effect on system behavior; Clark and Mangel 2001).

In the context of management and conservation, there are at least three other sources of uncertainty that can influence decision thresholds and that should thus be noted: partial observability, partial controllability and structural uncertainty (Williams et al. 1996, 2002, Johnson et al. 1997, Conroy et al. 2003). Partial observability occurs when state variables (e.g., $\psi_{t}$ in the example of species A) are measured with error. This form of uncertainty is due to sampling variation associated with the estimation of the state variable(s) of interest. Partial controllability results from the inability to accurately implement specified management actions and from imprecise translation of management actions into effects on the system. Structural uncertainty, also called model uncertainty (Burgman 2005), reflects the incomplete understanding of system behavior and is frequently dealt with through the simultaneous consideration of multiple models. For the sake of simplicity, we restricted our illustration of the effect of uncertainty on decision thresholds to the treatment of environmental stochasticity (Numerical example 1) and structural uncertainty (Numerical example 2, see next section). We focused on these two sources of uncertainty because they are particularly easy to understand but also because they can easily be incorporated into the SDM process (whereas this is much more difficult for partial observability). Several studies have explored the importance of these different sources of uncertainty on the decision making process in the context of resource management (e.g., Williams et al. 1996)

\section{Thresholds and Adaptive Management}

In the preceding sections, we described a method to derive decision thresholds when a single model is thought to provide a good approximation to system behavior. However, many real world situations are characterized by structural uncertainty, reflecting an 
incomplete understanding of system behavior. In such situations, several hypotheses (each hypothesis can be represented mathematically by a model) are put forward to explain the behavior of the system of interest. When more than one model is considered, decisions can be based on the weighted average of the dynamics predicted by the different models. Model weights (i.e., probabilities reflecting the relative degrees of faith in the predictions of the different models) can be assigned to all candidate models. These model weights characterize the perceived relative abilities of the different models to predict changes in system state following the implementation of specified management decisions. Sometimes SDM is applied to one-time decisions (e.g., land purchases), whereas at other times we face sequential decision processes in which a decision is repeated periodically (e.g., ongoing habitat management). For both one-time processes and the beginning of sequential SDM processes, model weights can be based either on inference from analyses of historical data or on political expediency (e.g., equal weights give no perception of advantage to a particular model).

Regardless of the origin of initial model weights, sequential processes provide an opportunity to learn about the predictive abilities of the different models. Let $p_{i}(t)$ denote the weight associated with model $m_{i}$ at time $t$, where

$$
\sum_{i=1}^{n} p_{i}(t)=1
$$

and $n$ is the total number of models. Bayes' Theorem provides a means to update model weights (Williams et al. 2002):

$$
p_{i}(t+1)=\frac{p_{i}(t) \times P_{i}\left(x_{t+1} \mid x_{t}, d_{t}\right)}{\sum_{i=1}^{n} p_{i}(t) \times P_{i}\left(x_{t+1} \mid x_{t}, d_{t}\right)}
$$

where $P_{t}\left(x_{t+1} \mid x_{t}, d_{t}\right)$ is the probability of the observed state at $t+1$ under model $m_{i}$, given that the system was in state $x_{t}$ at time $t$ and that decision $d_{t}$ was implemented. Updating is then a function of the model weight or prior probability at time $t$, reflecting accumulated knowledge, and the new information about how well the model predicted the state transition between $t$ and $t+1$. These updated probabilities then become the new model weights (or new priors) for the next decision and set of predictions (Kendall 2001, Nichols 2001, Williams et al. 2002). Provided that reasonable models have been included in the model set, this iterative process should lead to the identification (high model weights) of models that provide good predictions. This application of SDM to sequential decision processes is typically referred to as adaptive management (Walters 1986, Williams et al. 2002) and provides decision thresholds at each decision point and also improves knowledge of the ecological system (including ecological thresholds). There is some ability
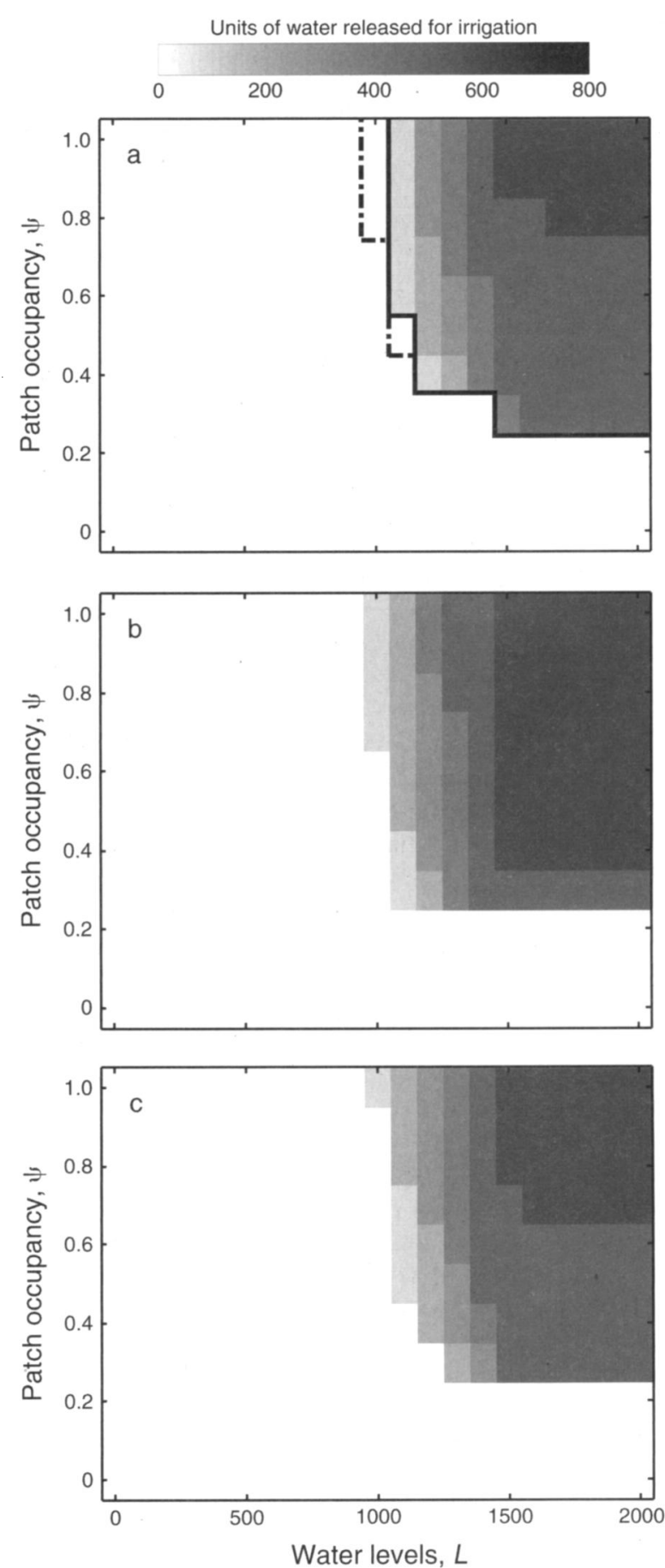

FIG. 4. Plots of optimal irrigation decisions as a function of water levels and patch occupancy $(\psi)$ of species A for (a) Model 1 , (b) Model 2, and (c) assuming equal weights for Model 1 and Model 2. Here the standard deviation associated with parameter $\beta$ in Eq. $7, \operatorname{SD}(\beta)$, for Model 1 and Model 2 was set to 0.001 . The shades of gray correspond to the amount of water released for irrigation (from 0 water units [lighter shade] to 800 units [darker shade]).The thick black line in panel (a) indicates the decision threshold at the boundary between some irrigation and no irrigations. The dot-dashed line in panel (a) indicates a shift in the decision threshold when compared to Fig. 2a [in Fig. $2 \mathrm{a}, \mathrm{SD}(\beta)=0$ ]. 


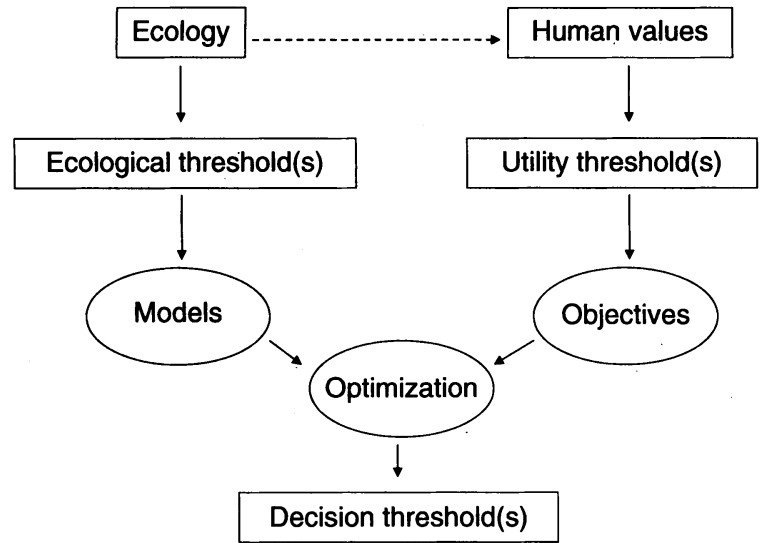

Fig. 5. Flow chart showing the relationship among the different types of thresholds. Ecological thresholds are determined by our understanding of the ecology of the system and are incorporated into models of system behavior. Utility thresholds are determined subjectively and reflect stakeholder values (although in some circumstances these values can be based on knowledge of the ecology of the system, as indicated by the dashed arrow). Decision thresholds are conditional on and derived from ecological and utility thresholds.

of this adaptive management process to accommodate situations in which system behavior and associated ecological thresholds change over time. However, this ability requires that the timescale of changes in system behavior be large relative to the timescale of the decision process itself (Williams et al. 2007).

When more than one model is considered for sequential decision processes, two approaches can be applied: passive or active adaptive management. Optimization algorithms for passive adaptive management treat the model weights as fixed values that remain constant over the time frame of the optimization. Learning about the system behavior occurs, but as a byproduct of management (Williams et al. 2002). By contrast, in active adaptive optimization the model weights are included as an information state during the optimization process (Williams 1996). Thus, active adaptive management is a formal attempt to deal with the so-called dual control problem of simultaneously meeting short-term system objectives and learning in order to make even better decisions in the future (Williams et al. 2002). The illustrative example presented next uses passive adaptive optimization algorithms, but both passive and active adaptive optimizations are implemented in the program ASDP (Lubow 2001).

\section{Numerical example 2}

In the preceding example we determined decision thresholds with only one model at a time. Now suppose that there are two prevailing hypotheses about how the system works, but that current scientific knowledge is equivocal about which hypothesis better conforms to reality. Under the first hypothesis, patch colonization is only possible when water levels reach 1500 units of water (this corresponds to Model 1 described earlier), whereas under the second hypothesis patch colonization can occur at 800 units of waters (this corresponds to Model 2 described earlier). Decision thresholds obtained from single-model optimization for Model 1 and Model 2 are presented in Fig. 4a, b, respectively (these models assumed $\operatorname{SD}(\beta)=0.001$ ). These results emphasize the points that knowledge of the nature of this ecological threshold is indeed relevant to management, and that the reduction of structural uncertainty is likely to lead to better management.

The ability to consider multiple models in decisionmaking provides a transparent means of dealing with competing hypotheses about system behavior. If no previous data tend to support one model more than the other, equal weights can be assigned to each model (Kendall 2001). We computed decision thresholds using a passive adaptive optimization algorithm with equal weight assigned to Model 1 and Model 2, and the resulting decision thresholds are represented in Fig. 4c. Not surprisingly, the decision thresholds resulting from the passive adaptive optimization were intermediate between the ones obtained from the single-model optimizations of Model 1 and Model 2 (Fig 4). Note that in the case of the passive adaptive optimization we have to rerun the optimization at every time period to revise the decision. Thus, at each time 'step predictions from each model can be confronted with the monitoring data and new weights can be computed. As the weights assigned to each model evolve over time (i.e., as we learn), the decision thresholds will also change over time.

\section{Discussion}

The above examples were intended to illustrate the SDM process and to highlight the roles of the three defined classes of thresholds in that process. Indeed, the SDM framework specifies an unambiguous context within which the three kinds of thresholds, and their respective roles in management, can be understood. Based on our operational definitions, ecological thresholds are incorporated into system models that are used to project the consequences of management actions to system state variables. The identification and modeling of such thresholds are important components of the science associated with conservation, in general, and SDM, in particular. In contrast, utility thresholds are subjectively determined and reflect stakeholder values by specifying desirable and undesirable values of system state variables. Utility and ecological thresholds may coincide, but there is no necessary relationship between them. Finally, we have defined decision thresholds as derivative of the other components of the decision process (Fig. 5). Under the SDM process that we outlined above, decision thresholds are not determined subjectively or arbitrarily, as is common to many current approaches to conservation. Instead, they can be based on optimization algorithms, with specific values condi- 
tional on stated objectives, available management actions, and the models of system behavior. Thus, while ecological and utility thresholds may be completely unrelated, decision thresholds are related in a very formal way to these other two types of thresholds. We make no claim that our framework provides the only possible way of viewing thresholds. However, we do claim that it is logical, consistent and can be proven to yield optimal management decisions conditional on the current state of knowledge (in this case the models and their respective weights represent our current state of knowledge).

With respect to ecological thresholds, SDM provides a way to judge the relative importance of different thresholds to management. Wiens et al. (2002) noted that "nature is full of thresholds layered upon thresholds" (reviewed in Bestelmeyer 2006). Thus, one temptation may be for biologists to become distracted by attempting to identify and measure thresholds that may be scientifically interesting, but not necessarily of high conservation significance. However, by stating clear conservation objectives and potential management actions, and then focusing on models of system response to those actions, conservation biologists can focus on the ecological thresholds that are most relevant to use of those actions to achieve objectives. Conveniently, when applied to sequential decision processes the SDM framework also enables investigators to learn about ecological threshold(s) at each iteration of the process. New information about ecological thresholds can be incorporated into models of system behavior, which in turn can help improve the management of the system. Furthermore, there is some ability of this adaptive management process to accommodate situations in which system behavior and associated ecological thresholds change over time. Of course as with any other approach to management decisions, SDM can only work when the state variables of the managed system are within controllable bounds of the state space. For example, in the case of fisheries management if the management goal is to control an invasive species, and if the management action consists of harvesting the invasive species, there may be a limit beyond which the population of invasive becomes uncontrollable with respect to the available potential management actions (Zipkin et al. 2008).

One common misconception is that a system, with its various ecological thresholds, must be well understood before one can develop useful models for conservation purposes (see also Clark and Mangel 2001). This misconception can lead to calls for additional monitoring and information that frequently represent inefficient uses of conservation funds (Nichols and Williams 2006). SDM can be applied even when little information is available about system behavior. All that is required is some basis for making predictions about effects of management actions on managed systems, a logical requirement of any type of informed management. The approach provides a way to apply available knowledge to manage the system at present and to increase this knowledge for better management in the future (Williams et al. 2007). Uncertainty about system behavior can always be incorporated in the models. Most important management decisions simply should not be postponed indefinitely, especially given that making no decision is itself a decision, and SDM focuses on making decisions based on available knowledge of system behavior and responses to management actions. Thus, the approach provides managers with a defensible set of policies. We note that in some cases SDM is built upon expert opinions (and perhaps competing opinions), but in a logical, transparent and rigorous way.

To conclude, SDM can be very beneficial to conservation by adding transparency to the process that produces decision thresholds. Given that the concept of threshold has now entered the public arena and is even used by the U.S. Congress to discuss natural resource issues (Bestelmeyer 2006), it is essential that the different kinds of thresholds and their respective roles in decision processes be clearly understood by those involved in the processes. Similarly, consideration of SDM clarifies the respective roles of policy makers and scientists. The SDM framework distinguishes the components of the decision process that are inherently subjective (objectives and management actions) from those that are more objective (models of system behavior, estimates of system state and analytical methods to derive decision thresholds). This distinction draws a boundary between the components that are driven primarily by value judgments and the components that are more in the domains of ecological and management science. Clarifying the role of scientists and policy makers when setting thresholds for conservation could prove to be a valuable step toward the implementation of better conservation decisions.

\section{ACKNOWLEDGMENTS}

The authors thank the U.S. Geological Survey Status and Trends of Biological Resource Program for funding this project. We are particularly grateful to Paul Geissler for sponsoring and coordinating funding for this research. We appreciate the comments from Evan Cooch, Michael Conroy, Larissa Bailey, Beth Gardner, Sarah Haas, Allan O'Connell, Ken Williams, and one anonymous reviewer.

\section{Literature Cited}

Bellman, R. 1957. Dynamic programming. Princeton University Press, Princeton, New Jersey, USA.

Bennetts, R. E., J. E. Gross, K. Cahill, C. McIntyre, B. B. Bingham, A. Hubbard, L. Cameron, and S. L. Carter. 2007. Linking monitoring to management and planning: assessment points as a generalized approach. George Wright Forum 24:59-77.

Benton, T. G. 2003. Understanding the ecology of extinction: are we closer to the critical threshold? Annales Zoologici Fennici 40:71-80.

Bestelmeyer, B. T. 2006. Threshold concepts and their use in rangeland management and restoration: the good, the bad and the insidious. Restoration Ecology 14:325-329. 
Bodin, P., and B. L. B. Wiman. 2007. The usefulness of stability concepts in forest management when coping with increasing climate uncertainties. Forest Ecology and Management 242: 541-552.

Brown, J. H., T. J. Valone, and C. J. Curtin. 1997. Reorganization of an arid ecosystem in response to recent climate change. Proceedings of the National Academy of Sciences (USA) 94:9729-9733.

Burgman, M. 2005. Risks and decisions for conservation and environmental management. Cambridge University Press, Cambridge, UK.

Burnham, K. P., and G. C. White. 2002. Evaluation of some random effects methodology applicable to bird ringing data. Journal of Applied Statistics 29:245-264.

Caughley, G. 1994. Directions in conservation biology. Journal of Animal Ecology 63:215-244.

Clark, C. W., and M. Mangel. 2001. Dynamic state variable models in ecology. Oxford University Press, New York, New York, USA.

Clemen, R. T., and T. Reilly. 2001. Making hard decisions with decision tools. Duxbury Press, Pacific Grove, California, USA.

Conroy, M. J., C. R. Allen, J. T. Peterson, L. J. Pritchard, and C. T. Moore. 2003. Landscape change in the Southern Piedmont: challenges, solutions, and uncertainty across scales. Conservation Ecology 8:3.

Conroy, M. J., and C. T. Moore. 2001. Simulation models and optimal decision making in natural resource management. Pages 91-104 in T. M. Shenk and A. B. Franklin, editors Modeling in natural resource management: valid development, interpretation and application. Island Press, Washington, D.C., USA.

Dorazio, R. M., and F. A. Johnson. 2003. Bayesian inference and decision theory: a framework for decision making in natural resource management. Ecological Applications 13: $556-563$.

Fahrig, L. 2001. How much habitat is enough? Biological Conservation 100:65-74.

Field, S. A., A. J. Tyre, N. Jonzen, J. R. Rhodes, and H. P Possingham. 2004. Minimizing the cost of environmental management decisions by optimizing statistical thresholds. Ecology Letters 7:669-675.

Fonnesbeck, C. J. 2005. Solving dynamic wildlife resource optimization problems using reinforcement learning. Natural Resource Modeling 18:1-39.

Gould, W. R., and J. D. Nichols. 1998. Estimation of temporal variability of survival in animal populations. Ecology 79 : 2531-2538.

Groffman, P. M., et al. 2006. Ecological thresholds: the key to successful environmental management or an important concept with no practical application? Ecosystems 9:1-13.

Gunderson, L. H. 2000. Ecological resilience in theory and application. Annual Review of Ecology and Systematics 31: 425-439.

Halpern, B. S., H. M. Regan, H. P. Possingham, and M. A McCarthy. 2006. Accounting for uncertainty in marine reserve design. Ecology Letters 9:2-11.

Holling, C. S. 1973. Resilience and stability of ecologica systems. Annual Review of Ecology and Systematics 4:1-23.

Huggett, A. J. 2005. The concept of "ecological thresholds" in biodiversity and conservation. Biological Conservation 124 301-310.

Intriligator, M. D. 1971. Mathematical optimization and economic theory. Prentice-Hall, Englewood Cliffs, New Jersey, USA

Johnson, F. A., C. T. Moore, W. L. Kendall, J. A. Dubowsky, D. F. Caithamer, J. R. Kelley, and B. K. Williams. 1997.
Uncertainty and the management of mallard harvests. Journal of Wildlife Management 61:202-216.

Kendall, W. L. 2001. Using models to facilitate complex decisions. Pages 147-170 in T. M. Shenk and A. B. Franklin, editors. Modeling in natural resource management: valid development, interpretation and application. Island Press, Washington, D.C., USA.

Lande, R. 1987. Extinction thresholds in demographic models of territorial populations. American Naturalist 130:624-635.

Lubow, B. C. 1995. SDP: Generalized software for solving stochastic dynamic optimization problems. Wildlife Society Bulletin 23:738-742.

Lubow, B. C. 2001. Adaptive stochastic dynamic programming (ASDP): Version 3.2. Colorado State University, Fort Collins, Colorado, USA

MacKenzie, D. I., J. D. Nichols, J. A. Royle, K. H. Pollock J. E. Hines, and L. L. Bailey. 2006. Occupancy estimation and modeling: inferring patterns and dynamics of species occurrence. Elsevier, San Diego, California, USA.

Maxwell, D., and S. Jennings. 2005. Power of monitoring programmes to detect decline and recovery of rare and vulnerable fish. Journal of Applied Ecology 42:25-37.

McCarthy, M. A., and H. P. Possingham. 2007. Active adaptive management for conservation. Conservation Biology 21:956 963

Miranda, M. J., and P. L. Fackler. 2002. Applied computational economics and finance. MIT Press, Cambridge, Massachusetts, USA.

Nichols, J. D. 2001. Using models in the conduct of science and management of natural resources. Pages 11-34 in T. M Shenk and A. B. Franklin, editors. Modeling in natural resource management: development, interpretation and application. Island Press, Washington, D.C., USA.

Nichols, J. D., and B. K. Williams. 2006. Monitoring for conservation. Trends in Ecology and Evolution 21:668-673.

Pascual, M., and F. Guichard. 2005. Criticality and disturbance in spatial ecological systems. Trends in Ecology and Evolution 20:88-95.

Walters, C. J. 1986. Adaptive management of renewable resources. Macmillan Publishing Company, New York, New York, USA

Wiens, J. A., B. Van Horne, and B. R. Noon. 2002. Integrating landscape structure and scale into natural resource management. Pages 23-67 in J. Liu and W. W. Taylor, editors. Integrating landscape structure and scale into natural resource management. Cambridge University Press, New York, New York, USA

Williams, B. K. 1996. Adaptive optimization and the harvest of biological populations. Mathematical Biosciences 136:1-20.

Williams, B. K., F. A. Johnson, and K. Wilkins. 1996. Uncertainty and the adaptive management of waterfowl harvests. Journal of Wildlife Management 60:223-232.

Williams, B. K., and J. D. Nichols. 2001. System identification and the adaptive management of waterfowl in the United States. Wildlife Biology 7:223-236.

Williams, B. K., J. D. Nichols, and M. J. Conroy. 2002. Analysis and management of animal populations. Academic Press, San Diego, California, USA.

Williams, B. K., R. C. Szaro, and C. D. Shapiro. 2007. Adaptive management: The U.S. Department of the Interior Technical Guide. U.S. Department of the Interior, Washington, D.C., USA.

Zipkin, E. F., P. J. Sullivan, E. G. Cooch, C. E. Kraft, B. J. Shuter, and B. C. Weidel. 2008. Overcompensatory response of a smallmouth bass population to harvest: release from competition? Canadian Journal of Fisheries and Aquatic Sciences 65:2279-2292. 